\title{
Sorotipagem de amostras de Streptococcus suis isoladas de suínos em granjas dos Estados de São Paulo, Minas Gerais e Paraná ${ }^{1}$
}

\author{
Keila J.R. Pagnani ${ }^{2}$, Antonio F. Pestana Castro ${ }^{2,3^{*}}$, Marcelo Gottschalk ${ }^{4}$, Wanderley D. \\ Silveira $^{2}$ e Gerson Nakazato ${ }^{2}$
}

\begin{abstract}
Pagnani K.J.R., Pestana de Castro A.F., Gottschalk M., Silveira, W.D. \& Nakazato G. 2002. [Serotyping of Streptococcus suis strains isolated from pigs in the States of São Paulo, Minas Gerais e Paraná, Brazil.] Sorotipagem de amostras de Streptococcus suis isolados de suínos em granjas dos Estados de São Paulo, Minas Gerais e Paraná. Pesquisa Veterinária Brasileira 22(1):1-5. Depto Microbiologia e Imunologia, Instituto de Biologia, Universidade Estadual de Campinas (Unicamp), Campinas, SP 13081-970, Brazil.

Streptococcus suis infection in swine is common in all countries where hog production is well developed. This infection has been associated with bronchopneumonia, meningitis, arthritis, pericarditis, myocarditis, endocarditis, fibrinous polyserositis, septicaemia, rhinitis, and abortion. Streptococcus suis has also been described as a pathogen for ruminants and humans. In Brazil there are several clinical evidences about the existence of $S$. suis disease in pigs affecting more than 50\% of farms in States of São Paulo, Minas Gerais and Paraná. In the present research 51 strains of S. suis isolated from piggeries of the States of São Paulo, Minas Gerais and Paraná were collected from different pathologies such as septicaemia, meningitis, arthritis and pneumonia and been recovered either in pure culture or as the predominant organism from porcine tissues. Culture of specimens was carried out on $5 \%$ bovine blood agar plates incubated at $37^{\circ} \mathrm{C}$ for $24 \mathrm{hr}$. For the biochemical identification the a-hemolytic colonies of all capsulated isolates were submitted to various conventional tests, such as hydrolysis of arginine, Voges-Proskauer Test, and production of acid from various carbohydrates (inulin, salicin, trehalose, lactose, sucrose, sorbitol, mannitol and glycerol). The strains were also tested for their ability to grow in the presence of $6,5 \% \mathrm{NaCl}$ and for the amylase production. In addition strains were tested by Api Strep 20 to confirm the identification of S.suis. For capsular typing only capsulated strains were typed by co-agglutination test, using antisera raised in rabbits against all reference strains from serotypes 1 to 8 . Strains belonging to other serotypes were also typed. The co-agglutination was used for serotyping and the capsular reaction test was carried out for measuring the potency of the prepared antisera. From the total of 51 examined strains the following results were obtained, with regard to serotyping: $30(58,8 \%)$ were serotype $2,11(21,6 \%)$ were serotype 3 , seven $(13,72 \%)$ were serotype 7 , two $(3,92 \%)$ were serotype 1 and one strain belonged to serotype $14(1,96 \%)$. As far as we are concerned, this is the first report on the isolation of a large number of $S$. suis strains in Brazil, from cases of illness caused by this bacterium among piglets. Also it was carried out serotyping of the isolates, showing a high prevalence of serotype 2 , as compared to other known serotypes of $S$. suis.
\end{abstract}

INDEX TERMS: Streptococcus suis, isolation, serotyping, co-agglutination.

\footnotetext{
${ }^{1}$ Aceito para publicação em 27 de novembro de 2001.

Trabalho realizado com subsídios da FAPESP e do CNPq.

${ }^{2}$ Instituto de Ciências Biomédicas, Universidade de São Paulo, SP. E-mail: apestana@icb.usp.br. *Autor para correspondência.

${ }^{3}$ Instituto de Biologia, Departamento de Microbiologia e Imunologia, Unicamp, Campinas, SP. E-mail: kjordao@obelix.unicamp.br

${ }^{4}$ Escola de Medicina Veterinária de Saint-Hyacinthe (GREMIP), Quebec, Canadá. E-mail: gottschm@medvet.umontreal.ca
}

RESUMO.- Infecções causadas por Streptococcus suis são muito comuns em países onde a indústria de carne suína é desenvolvida. Estas infecções estão relacionadas a casos clínicos de broncopneumonia, meningite, artrite, pericardite, miocardite, endocardite, poliserosite fibrinosa, septicemia, rinite e aborto. Esta bactéria também foi descrita como patógeno de ruminantes e humanos. No Brasil há evidências clínicas da existência de processos infecciosos causados por S. suis afe- 
tando mais de 50\% das granjas em Estados como São Paulo, Minas Gerais e Paraná. No presente estudo foram isoladas 51 amostras de $S$. suis de granjas do Estados acima referidos, coletadas de diferentes casos clínicos como septicemia, meningite, artrite e pneumonia, tendo sido obtidas ou em cultura pura ou como patógeno de maior predominância nos tecidos de suínos. Este material foi semeado em Columbia ágar sangue adicionado de $5 \%$ de sangue bovino e incubado a $37^{\circ} \mathrm{C}$ por 24 horas. Para a identificação bioquímica as colônias que apresentavam a-hemólise, bem como as amostras padrão, foram submetidas a testes convencionais para a confirmação da espécie $S$. suis, tais como: hidrólise de arginina, teste de Voges-Proskauer, e produção de ácido a partir de vários carboidratos (inulina, salicina, trealose, lactose, sacarose, sorbitol, manitol e glicerol). As amostras também foram testadas para habilidade de crescimento em meio de TSA com $6,5 \%$ de $\mathrm{NaCl}$ e para a produção de amilase. Todas as amostras que fizeram parte desta pesquisa foram testadas pelo sistema Api 20 Strep para confirmação dos resultados obtidos nos testes convencionais. Para a sorotipagem foram produzidos antissoros de 1 a 8 . Outras amostras não pertencentes a estes sorotipos também foram sorotipadas. 0 antissoro produzido em coelhos foi titulado pelo teste de aglutinação em tubo com 2-mercaptoetanol e pelo teste de reação capsular e, quando adequados, foram usados no teste de co-aglutinação, para a sorotipagem das amostras de $S$. suis. A sorotipagem das 51 amostras isoladas mostraram os seguintes resultados: $30(58,8 \%)$ foram classificadas como sorotipo 2, $11(21,6 \%)$ das amostras como sorotipo 3 , sete $(13,72 \%)$ como sorotipo 7 , duas $(3,92 \%)$ como sorotipo 1 e uma amostra como pertencente ao sorotipo14 (1,96\%). Este é o primeiro relato do isolamento de um grande número de amostras de $S$. suis no Brasil, de casos típicos de processos infecciosos causados por esta bactéria. Também foi realizada a sorotipagem dos isolados, mostrando uma alta prevalência do sorotipo 2 , quando comparada com a dos demais sorotipos encontrados.

TERMOS DE INDEXAÇÃO: Streptococcus suis, isolamento, sorotipagem, co-aglutinação.

\section{INTRODUÇÃO}

Um novo grupo de estreptococos a-hemolítico oriundo de infecções em suínos foi caracterizado, pela primeira vez, bioquímica e sorologicamente, por de Moor entre 1956 e 1963, como novos grupos de Lancefield R, S, e T (De Moor 1963).

A designação Streptococcus suis como uma nova espécie bacteriana foi oficializada por Kilper-Balz \& Schleifer (1987).

Em 1975, Windsor \& Elliot (1975) isolaram outros estreptococos provenientes de suínos que correspondiam ao grupo R de Moor e o nomearam de $S$. suis tipo 2. 0 tipo 1 estava associado a quadros de meningite em suínos recém nascidos e o tipo 2 ocorria em qualquer faixa etária. Amostras isoladas e que reagiam com ambos os antissoros: tipo 1 e tipo 2, foram designadas tipo capsular 1/2. Entre 1983 e 1995, um total de 32 novos tipos capsulares havia sido descrito, completando 35 sorotipos (Perch et al.1983; Higgins et al.
1995). As amostras padrão isoladas foram obtidas de suínos com quadros clínicos compatíveis de infecção por $S$. suis, com exceção do tipo capsular 14, isolado de humanos, tipos $17,18,19$, e 21 isolados de suínos saudáveis e os tipos 20 e 31 de bovinos com quadro de septicemia e o tipo 33 de ovelhas, com mesma sintomatologia (Gottschalk et al.1989). Jansen \& Van Dorssen (1951) descreveram meningites em suínos causadas por S. suis na Noruega. Field et al. (1954) também relataram casos de meningites e artrite em leitões na Inglaterra, ocasionados por esta bactéria. Desde então o $S$. suis tem sido descrito em todos os países, onde a indústria de carne suína tem expressão, tanto em granjas de manejo tradicional quanto em granjas de criação intensiva.

Em quase todos países o sorotipo mais comumente isolado é o sorotipo 2. A maioria das amostras de $S$. suis isoladas pertence a um número restrito de sorotipos normalmente compreendidos entre o sorotipo 1 a 8 (Galina et al.1992, Kataoka et al. 1993, Prieto et al. 1994, Higgins \& Gottschalk 1996, Hogg et al.1996, Reams et al.1996).

No Brasil, salvo alguns isolamentos esporádicos do sorotipo 2 , que não foram relatados sob a forma de publicação consistente, ao que se sabe, nada foi encontrado na literatura disponível.

\section{MATERIAL E MÉTODOS}

\section{Amostras bacterianas}

As amostras padrão do sorotipo 1 a 8 de $S$. suis, pertencentes à bacterioteca da Faculdade de Medicina Veterinária de Saint Hyacinthe, Universidade de Montreal, Quebec, Canadá, foram utilizadas na produção de antissoros. Este estudo incluiu 51 amostras isoladas e provenientes de casos de septicemia, endocardite, pneumonia e artrite de granjas dos Estados de São Paulo, Paraná e Minas Gerais.

\section{Isolamento e identificação}

A coleta das amostras bacterianas foi feita a partir de suabes estéreis de meninges, tonsilas, articulações, coração e outros órgãos internos, de animais doentes submetidos à necrópsia. Os materiais foram semeados em placas de Columbia ágar sangue a 5\% de sangue bovino, examinadas e subcultivadas em meio líquido de Todd-Hewitt (Difco Laboratories, USA). Para as provas de identificação bioquímica tomou-se uma colônia da amostra em estudo crescida em meio sólido, efetuando-se os seguintes testes: produção de amilase, teste de Voges-Proskauer (VP), reação de vermelho de metila (VM), crescimento em TSA com $6,5 \%$ de $\mathrm{NaCl}$ e produção e ácidos a partir de vários carboidratos (inulina, salicina, trealose, lactose, sacarose, sorbitol e manitol). Os isolados foram considerados como S. suis, quando os seguintes resultados foram obtidos: VP negativo, VM positivo, nenhum crescimento em meio de TSA $+6.5 \% \mathrm{NaCl}$, produção de amilase positiva, e quando ocorria fermentação da trealose. Todas as amostras utilizadas no presente estudo foram ainda testadas pelo sistema Api 20 Strep (Bio-Mérieux) para confirmação dos resultados obtidos nos testes convencionais.

\section{Identificação sorológica}

Foram preparados antissoros contra os sorotipos de 1 a 8 descritos anteriormente. Quanto a amostras pertencentes a outros sorotipos, a sorotipagem foi realizada na Faculdade de Medicina Veterinária de Saint-Hyacinthe, Quebec, Canadá. Para a produção de antissoro as amostras padrão foram semeadas em placas de Columbia ágar com $5 \%$ de sangue bovino por 18 horas a $37^{\circ} \mathrm{C}$ adicionado de 


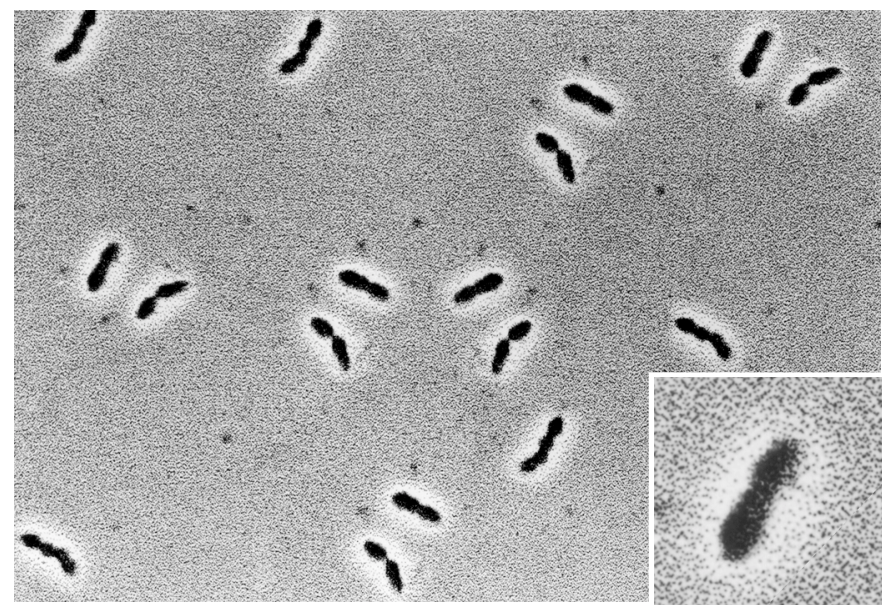

Fig. 1. Teste de Reação Capsular - Streptococcus suis sorotipo 2, para aferição do antissoro preparado para as reações de coaglutinação, com a finalidade de tipificar este sorotipo entre as amostras isoladas.

suplemento SR-126 (Oxoid). Os antissoros foram produzidos a partir de inoculação de antígeno em coelhos albinos com $3 \mathrm{~kg}$ de peso vivo em 3 doses semanais com concentrações crescentes de bactéria durante quatro semanas. Dez dias após a última inoculação foram obtidas amostras de sangue e a resposta sorológica foi avaliada pelo teste de reação capsular (Higgins et al. 1995), conforme representado na Fig.1. Os antissoros foram submetidos a teste de aglutinação em tubo com 2-mercaptoetanol e somente aqueles que apresentaram título de 45 ou mais é que foram utilizados no teste de co-aglutinação (Mittal et al. 1984). O preparo dos reagentes para o teste de coaglutinação foi realizado segundo Mittal et al. (1984). Os resultados dos testes foram descritos semiquantitativamente como: $0,1+, 2+$, $3+$, e $4+$. Somente reações de $2+$ foram consideradas positivas. Em todos os testes foram incluídos controles positivos e negativos. $\mathrm{O}$ controle positivo foi representado por cultura de uma amostra de

Quadro 1. Porcentagem de amostras positivas em 11 testes bioquímicos convencionais com 51 amostras de Streptococcus suis isoladas no Brasil e comparação com os resultados esperados em cada teste para os sorotipos de 1 a 8

\begin{tabular}{|c|c|c|}
\hline Testes bioquímicos & $\begin{array}{c}\text { Resultados esperados } \\
\text { para os sorotipos } 1 \text { a } 8\end{array}$ & $\begin{array}{c}\text { Porcentagem de amostras } \\
\text { positivas }(\%)\end{array}$ \\
\hline Acetoína(VP) a & b & 0 \\
\hline Arginina & $(+)^{c}$ & 90 \\
\hline Rafinose & - & 21,6 \\
\hline Inulina & $(+)^{c}$ & 86,3 \\
\hline Lactose & + & 100 \\
\hline Manitol & - & 100 \\
\hline $\mathrm{TSA}+6,5 \% \mathrm{NaCl}{ }^{\text {a }}$ & - & 100 \\
\hline Salicina ${ }^{\mathrm{a}}$ & + & 100 \\
\hline Sorbitol & - & 100 \\
\hline Sacarose & + & 100 \\
\hline Trealose $^{a}$ & + & 100 \\
\hline Amilase $^{\mathrm{a}}$ & + & 100 \\
\hline
\end{tabular}

Testes usados em nosso laboratório como identificação presuntiva.

b+ Positivo, - negativo.

${ }^{\mathrm{C}}$ Resultados variáveis.

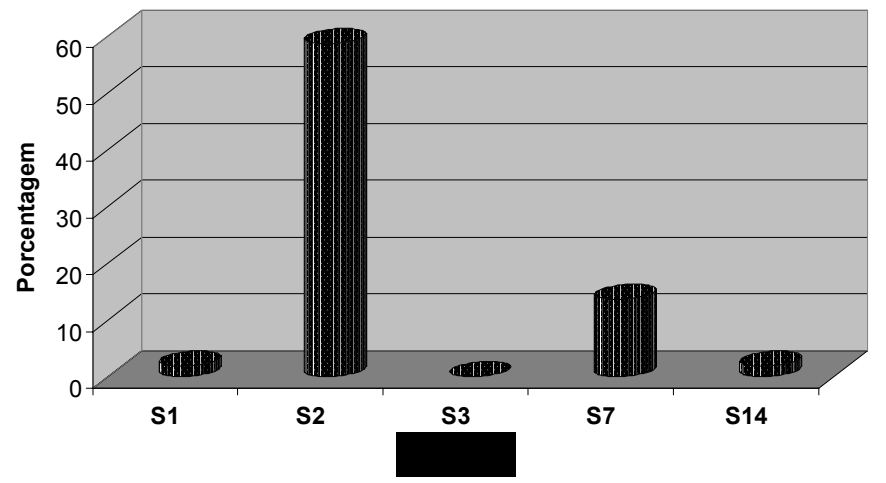

Fig. 2. Porcentagem de amostras Streptococcus suis sorotipadas, entre os 51 isolados de materiais clínicos de suínos com sintomatologia de infecção por este microrganismo, coletados de granjas das cidades de Bragança Paulista (SP), Castro (PR) e Patrocínio (MG).

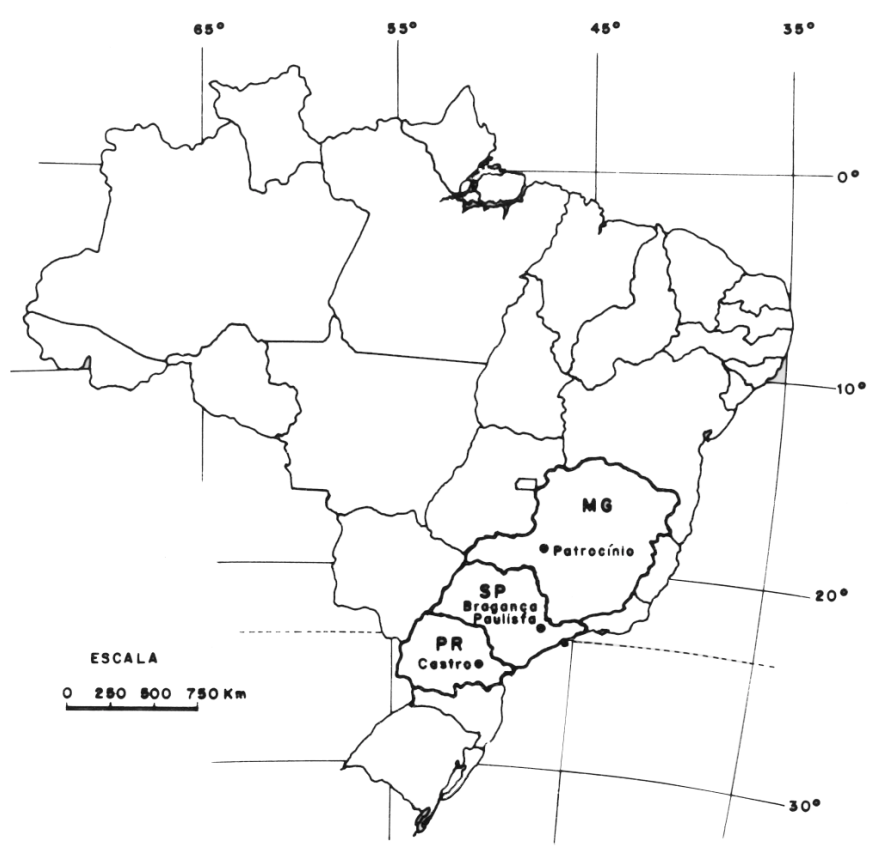

Fig. 3. Mapa do Brasil ilustrando as cidades de cada estado de onde foram isoladas as 51 amostras de Streptococcus suis.

Enteroccoccus faecalis (ATCC 19433) pertencendo ao grupo D de Lancefield e o controle negativo por uma suspensão de células de Staphylococcus aureus sensibilizado com soro de coelho normal.

\section{RESULTADOS}

Os resultados dos testes bioquímicos das 51 amostras de $S$. suis isoladas estão apresentados no Quadro 1, observando-se reação negativa para VP, positiva para a produção de amilase e negativa para crescimento em meio de TSA, contendo 6,5\% de $\mathrm{NaCl}$. Estes dados, além dos resultados de fermentação para os carboidratos lactose, manitol, salicina, sorbitol, sacarose e trealose estão de acordo com a literatura 
(Gottschalk et al. 1991) para a identificação bioquímica de $S$. suis. No mesmo quadro se encontram resultados variáveis para alguns poucos testes, a saber arginina, rafinose e inulina, que são descritos para S. suis (Gottschalk et al. 1991). Foi utilizado ainda o sistema de identificação API Strep 20 (Bio-Mérieux) para comparação com os resultados obtidos com os testes bioquímicos convencionais. Nenhum padrão bioquímico pode ser associado a algum sorotipo específico. Com relação à fermentação de inulina 13,7\% das amostras de sorotipo 7 foram negativas; com relação a rafinose apresentou $21,6 \%$ das amostras foram positivas, resultados compatíveis com o citado acima, em relação a estes carboidratos.

Das 51 amostras isoladas e sorotipadas das regiões de São Paulo, Minas Gerais e Paraná 30 pertenciam ao sorotipo $2(50,8 \%), 11$ amostras $(21,5 \%)$ ao sorotipo 3 , sete amostras $(13,7 \%)$ ao sorotipo 7 , duas amostras $(3,9 \%)$ ao sorotipo 1 e uma amostra $(1,9 \%)$ ao sorotipo 14 (Fig. 2).

$\mathrm{Na}$ Fig. 3 estão representados os estados brasileiros e respectivas cidades de onde foram isoladas as 51 amostras de $S$. suis. Em dados não publicados foi verificado que das 30 amostras pertencentes ao sorotipo 2 , em que pese a distância relativamente grande entre as cidades, com exceção de uma amostra, todas as demais foram enquadradas num mesmo clone (distribuição clonal) de acordo com o teste de "Random Amplified Polymorphic" DNA (RAPD) (Sonia Chatellier, Faculdade de Medicina Veterinária de Saint-Hyacinthe, Canadá, comunicação pessoal).

\section{DISCUSSÃO}

No presente estudo foram isoladas amostras de Streptococcus suis pertencentes aos sorotipos de 1 a 8 . Estes sorotipos são relatados na literatura como os que apresentam maior frequiência nos países onde a doença e respectivo agente causal têm sido encontrados. Com relação às provas bioquímicas convencionais os resultados foram satisfatórios sendo que as amostras apresentaram as reações descritas na literatura para S. suis (Gottschalk et al. 1991). Como recomendado por estes últimos autores foram utilizados 5 testes para a identificação presuntiva de $S$. suis, a saber: crescimento negativo em TSA $+6,5 \%$ de $\mathrm{NaCl}$, teste de Voges-Proskauer (VP) negativo, reação de amilase positiva e produção de ácido a partir de trealose e salicina (Quadro 1) como indicado por Higgins \& Gottschalk (1990). As amostras estudadas apresentaram $100 \%$ de negatividade para a fermentação de sorbitol e manitol, embora já tenham sido isoladas de ruminantes amostras positivas para estes carboidratos (Hommez et al. 1988). Algumas amostras $(13,7 \%)$ apresentaram fermentação da inulina negativa, mas estariam relacionadas às exceções que ocorrem para esta prova. Os casos atípicos para as provas bioquímicas sempre ocorrem e somente a sorotipagem pode resolver estas discrepâncias.

$\mathrm{Na}$ sorotipagem as amostras foram classificadas como pertencentes aos sorotipos 1, 2, 3, 7 e 14. Das 51 amostras isoladas, 30 pertenciam ao sorotipo $2(50,8 \%), 11$ amostras $(21,5 \%)$ ao sorotipo 3 , sete amostras $(13,7 \%)$ pertenciam ao sorotipo 7, duas amostras $(3,9 \%)$ ao sorotipo 1 , e uma amos- tra $(1,9 \%)$ ao sorotipo 14 . Vale ressaltar a importância do isolamento de uma amostra pertencente ao sorotipo 14, pelo aspecto zoonótico já que foi descrito anteriormente por Arends \& Zanen (1988) e por Zanen \& Engel (1975) como agente etiológico de meningite em humanos.

A identificação correta deste patógeno é importante, bem como a sua respectiva classificação sorológica, haja vista a conotação já mencionada de alguns sorotipos com meningites e ou septicemia em suínos, como também a importância de alguns deles causando enfermidade em humanos, mormente meningite (Zanen \& Engel 1975, Arends \& Zanen 1988, Gottschalk et al. 1989).

Outro aspecto bastante interessante é que embora as amostras tenham sido isoladas de diferentes regiões brasileiras ilustradas na Fig. 3, mostrando distâncias consideráveis, ou sejam de $300 \mathrm{~km}$ ou mais, entre as cidades das quais as amostras do sorotipo 2 foram isoladas, foi observada uma distribuição clonal destes isolados quando submetidos ao teste de RAPD (Random polimorphic amplified DNA), comparando-os com diferentes iniciadores e amostras padrão de outros países (Sonia Chatellier, Faculdade Medicina Veterinária de Saint-Hyacinthe, Canadá, comunicação pessoal). Com base nestes achados e pela distância entre as cidades das quais as amostras foram isoladas, poderíamos supor que todas tenham tido uma amostra ancestral comum, no caso do Brasil, no que concerne às regiões estudadas.

Porém, como se tratam de estudos preliminares em nosso país, enfocando apenas materiais isolados de três cidades dos Estados de São Paulo, Paraná e Minas Gerais, seria preciso isolar um número maior de amostras, provenientes de outras regiões. Assim, seria possível ter-se uma idéia mais abrangente da distribuição da bactéria em nosso meio e melhor compreender a sua relação com enfermidades em suínos no que diz respeito a $\mathbf{S}$. suis como importante agente zoonótico na suinocultura brasileira. No que concerne a distribuição dos sorotipos isolados, esta pode mudar com o decorrer do tempo, dependendo da região em que é isolada a amostra (Gottschalk \& Segura 2000).

De qualquer modo, à semelhança do que ocorre em outros países, a presente pesquisa comprova a predominância do sorotipo 2 como causa de enfermidade em suínos, ressaltando ainda que outros sorotipos, em especial os sorotipos 3 e 7 foram relativamente frequientes em nossos achados.

Finalmente, chamamos a atenção para o fato que na literatura nacional consultada, este é o primeiro relato abrangente sobre a ocorrência de $S$. suis em nossas criações de suínos, em particular no que tange à prevalência do sorotipo 2 .

\section{REFERÊNCIAS}

Arends J.P. \& Zanen H.C.1988. Meningitis caused by Streptococcus suis in humans. Rev. Infect. Dis. 10:131-137.

De Moor C.E. 1963. Septicemic infections in pigs caused by haemolytic streptococci of new Lancefield groups designated $\mathrm{R}, \mathrm{S}$ and T. Antonie van Leeuvenhoek 29:272-280.

Field H.I., Buntain D. \& Done J.T. 1954. Studies on piglet mortality. I. Streptococcal meningitis and arthritis. Vet. Rec. 66:453-455. 
Galina L., Collins J.E. \& Pijoan C. 1992. Porcine Streptococcus suis in Minnesota. J. Vet. Diagn. Invest. 4:195-196.

Gottschalk M. \& Segura M. 2000. The pathogenesis of the meningitis caused by Streptococcus suis: the unresolved questions. Vet. Microbiol. 76:259272.

Gottschalk M., Higgins R., Jacques M., Mittal K.R. \& Henrichsen J. 1989. Description of 14 new capsular types of Streptococcus suis. J. Clin. Microbiol. 27:2633-2635.

Gottschalk M., Higgins R., Jacques M., Beaudin M. \& Henrichsen J. 1991. Characterization of six new capsular types (23 through 28) of Streptococcus suis. J. Clin. Microbiol. 29:2590-2594.

Higgins R. \& Gottschalk M. 1990. An update on Streptococcus suis identification. J. Vet. Diagn. Invest. 2:249-252.

Higgins R. \& Gottschalk M. 1996. Distribution of Streptococcus suis capsular types in 1995. Can. Vet. J. 37:242.

Higgins R., Gottschalk M., Boudreau M., Lebrun A. \& Henrichsen J.1995. Description of six new capsular types (29-34) of Streptococcus suis. J. Vet. Invest. 7:405-406.

Hogg A., Aamass S.F., Hoffman L.J., Wu C.C. \& Clark, L.K. 1996. A survey of Streptococcus suis isolations by serotype and tissue of origin. Proc. Am. Assoc. Swine Pract., 79-81.

Hommez J., Wullepit J., Cassimon P., Ceyssens K. \& Devries L.A. 1988. Streptococcus suis and other streptococcal species as a cause of extramammary infection in ruminants. Vet. Rec. 123:626-627.
Jansen E.J. \& Van Dorssen C.A. 1951. Meningoencephalitis bij varkens door streptococcen. Tijdschr. Diergeneeskd. 76:815-832.

Kataoka Y., Sugimoto C., Nakazawa M., Morozumi T. \& Kashiwazaki M. 1993. The epidemiological studies of Streptococcus suis infections in Japan from 1987 to 1991. J. Vet. Med. 55:623-626.

Kilper-Balz R. \& Schleifer K.H. 1987. Streptococcus suis sp. nov.; nom. rev. Int. J. Syst. Bacteriol. 37:160-162.

Mittal K., Higgins R., Larivière S. \& Leblanc D. 1984. A 2-mercaptoetanol tube agglutination test for diagnosis of Haemophilus pleuropneumoniae infection in pigs. Am J. Vet. Res. 45:715-719.

Perch B., Pedersen K.B. \& Henrichsen J. 1983. Serology of capsulated streptococci pathogenic for pigs: six new serotypes of Streptococcus suis. J. Clin. Microbiol. 17:993-996.

Prieto C., Garcia F.J., Suarez P., Imaz M. \& Castro J.M. 1994. Biochemical traits and antimicrobial susceptibility of Streptococcus suis isolated from slaughtered pigs. J. Vet. med. B. 41:608-617.

Reams R.Y., Harrington D.D., Glickman L.T., Thacker H.L. \& Bowersock T.L. 1996. Multiple serotypes and strains of Streptococcus suis in naturally infected swine herds. J. Vet. Diagn. Invest. 8:119-121.

Windsor R.S. \& Elliot S.D. 1975. Streptococcal infection in young pigs. IV. An outbreak of streptococcal meningitis in weaned pigs. J. Hyg. 75:6978 .

Zanen H.C. \& Engel H.W.B. 1975. Porcine streptococci causing meningitis and septicaemia in man. Lancet 7919:1286-1288. 